\title{
Antimatter production in proton-proton and heavy-ion collisions at ultrarelativistic energies
}

\author{
J. Cleymans, ${ }^{1}$ S. Kabana, ${ }^{2}$ I. Kraus, ${ }^{3}$ H. Oeschler, ${ }^{3,4}$ K. Redlich, ${ }^{5,6}$ and N. Sharma ${ }^{7}$ \\ ${ }^{1}$ UCT-CERN Research Centre and Department of Physics, University of Cape Town, Rondebosch 7701, South Africa \\ ${ }^{2}$ SUBATECH, 4 rue Alfred Kastler, F-44307 Nantes, France \\ ${ }^{3}$ Institut für Kernphysik, Darmstadt University of Technology, D-64289 Darmstadt, Germany \\ ${ }^{4}$ European Organization for Nuclear Research (CERN), Geneva, Switzerland \\ ${ }^{5}$ Institute of Theoretical Physics, University of Wroctaw, Pl-45204 Wroctaw, Poland \\ ${ }^{6}$ ExtreMe Matter Institute EMMI, GSI, D-64291 Darmstadt, Germany \\ ${ }^{7}$ Panjab University, Chandigarh, India
}

(Received 14 April 2011; revised manuscript received 12 August 2011; published 28 November 2011)

\begin{abstract}
One of the striking features of particle production at high beam energies is the near-equal abundance of matter and antimatter in the central rapidity region. In this paper we study how this symmetry is reached as the beam energy is increased. In particular, we quantify explicitly the energy dependence of the approach to matter-antimatter symmetry in proton-proton and in heavy-ion collisions. Expectations are presented also for the production of more complex forms of antimatter such as antihypernuclei.
\end{abstract}

DOI: 10.1103/PhysRevC.84.054916

PACS number(s): 25.75.Dw, 13.85.Ni

\section{INTRODUCTION}

One of the striking features of particle production at high energies is the nearly equal abundance of matter and antimatter in the central rapidity region [1,2]. It is believed that a similar symmetry existed in the initial stage of the universe. It remains a mystery how this symmetry got lost in the evolution of the universe, reaching a stage with no visible amounts of antimatter being present. Closely related to the matter-antimatter symmetry is the production of light antinuclei, hypernuclei, and antihypernuclei at high energies, especially in view of the recent observation of the anti- ${ }^{4} \mathrm{He}$ nucleus by the STAR Collaboration [3].

Since the first observation of hypernuclei in 1952 [4], there has been steady interest in searching for new hypernuclei and exploring the hyperon-nucleon interaction, which is relevant for nuclear physics (see, e.g., [5-8]). Hypernuclei decay with a lifetime that depends on the strength of the hyperon-nucleon interaction. While several hypernuclei have been found since the first observation, no antihypernucleus has ever been observed until the recent discovery of the antihypertriton in $\mathrm{Au}+\mathrm{Au}$ collisions at $\sqrt{s_{\mathrm{NN}}}=200 \mathrm{GeV}$ by the STAR Collaboration at the Relativistic Heavy Ion Collider (RHIC) [9]. The yield of (anti)hypernuclei measured by STAR is very large; in particular they seem to be produced with a yield similar to that of other (anti)nuclei, such as (anti- $)^{3} \mathrm{He}$. This abundance is much higher than that measured for hypernuclei and nuclei at lower energies [10]. It is of interest to understand the nature of this enhancement, and for this reason the systematics of antimatter production in high-energy hadron-hadron and heavy-ion collisions should be investigated.

The analysis of particle production to assess the degree of thermalization of the particle source has been undertaken over many decades [11-16]. It has been found that the thermalization assumption applies successfully to hadrons produced in a large number of particle and nuclear reactions at different energies [17-25]. This fact allowed the estimation of thermal parameters characterizing the particle source for each colliding system, which is relevant for the understanding of the thermal properties of dense and hot matter and for studies of QCD phase transitions [26,27].

In this paper, using the energy dependence of thermal parameters obtained from the statistical-thermal model analysis of particle yields in heavy-ion collisions [28,29], we present model estimates for (anti)hypernuclei multiplicity that can be directly compared to the recent results obtained in central $\mathrm{Au}+\mathrm{Au}$ collisions at RHIC. We discuss systematics of (anti)matter production at different energies. We also make predictions of (anti)matter and (anti)hypernuclei production at the Large Hadron Collider (LHC).

Recently, a very interesting analysis of the production of light nuclei, hypernuclei, and their antiparticles in central heavy-ion collisions was performed [30] within the statisticalthermal model. It was found that ratios of hypernuclei to $\Lambda$ particles exhibit an energy dependence similar to the $K^{+} / \pi^{+}$ ratio, with a clear maximum at low energy. The present work is considered to be an extension of the analysis performed in Ref. [30].

First, we compare the statistical-thermal model results for (anti)baryon production in heavy-ion and in proton-proton collisions. This, in general, requires knowledge of the energy dependence of thermal parameters in $p+p$ collisions, which is proposed in this paper based on recent data. In this context, we study quantitatively how the matter-antimatter symmetry is reached as the beam energy is increased for both $p+p$ and heavy-ion collisions. We also discuss the role of the strangeness content of particles and quantify different antibaryon/baryon ratios in $p+p$ and in heavy-ion collisions at Super Proton Synchrotron (SPS), RHIC, and LHC energies.

Second, we compare predictions of the statistical-thermal and coalescence models for different ratios of (anti)nuclei and (anti)hypernuclei in the context of recent STAR data obtained in central $\mathrm{Au}+\mathrm{Au}$ collisions at the top RHIC energy.

The paper is organized as follows: In Sec. II we discuss features of the statistical-thermal model. In Sec. III we 
compare the antibaryon/baryon ratios in $p+p$ and heavyion collisions and obtain the energy dependence of thermal parameters in $p+p$ collisions. We demonstrate the scaling behavior of the antibaryon/baryon ratios with their strangeness content. In Sec. IV we apply the thermal and the coalescence models to the production of nuclei and hypernuclei and their antiparticles. We also make predictions for (anti)nuclei and (anti)hypernuclei yield ratios at LHC energy. In Sec. V we summarize our results.

\section{THE STATISTICAL-THERMAL MODEL}

The statistical-thermal model assumes that in a highenergy collision at freeze-out all hadrons follow equilibrium distributions. The conditions at chemical freeze-out where inelastic collisions cease are given by the hadron abundances, while the particle spectra offer insight into the conditions at thermal freeze-out where elastic collisions cease. Once thermal parameters are fixed, the hadron-gas partition function gives all primordial thermodynamic observables of the system. The exact form of the partition function, however, depends on the statistical ensemble under consideration.

Within the grand-canonical (GC) ensemble, the quantum numbers of the system are conserved on average through the action of chemical potentials [24]. In other words, the baryon $B$, strangeness $S$, and charge content $Q$ are fixed on average by the $\mu_{B}, \mu_{S}$, and $\mu_{Q}$ chemical potentials, respectively. For each chemical potential one can introduce the corresponding fugacity $\lambda=e^{\mu / T}$, where $T$ is the temperature of the system.

In the GC ensemble the density of hadron species $i$ with mass $m_{i}$, quantum numbers $B_{i}, S_{i}$, and $Q_{i}$, and spin-isospin degeneracy factor $g_{i}$ is expressed through the second-order modified Bessel function $K_{2}(x)$ as

$$
n_{i}\left(T, \mu_{B}, \mu_{S}, \mu_{Q}\right)=\frac{g_{i}}{2 \pi^{2}} m_{i}^{2} T \lambda_{B}^{B_{i}} \lambda_{S}^{S_{i}} \lambda_{Q}^{Q_{i}} K_{2}\left(\frac{m_{i}}{T}\right) .
$$

The above form, valid in the Boltzmann approximation, is easily generalized to quantum statistics [28,29].

In the application of the statistical-thermal model the chemical potentials $\mu_{S}$ and $\mu_{Q}$ are typically constrained in the initial stage by the strangeness neutrality condition and by the fixed baryon-to-charge ratio. However, it is well established that the usual form of the statistical-thermal model formulated in the GC ensemble cannot be used when the temperature or the volume parameter $V$ or both are small [24,31]. As a general rule one needs $V T^{3}>1$ for a grand canonical description to hold [32-34]. This condition is not usually justified in $p+p$ collisions, requiring canonical (C) formulation of strangeness conservation. The exact strangeness conservation causes a suppression in particle ratios of strange (or multistrange) hadrons to pions or any strangeness neutral particles as compared to the corresponding ratio in the grand-canonical limit. The key parameter governing this effect can be quantified by the strangeness correlation volume [31].

\section{PRODUCTION OF ANTIBARYONS}

The chemical freeze-out conditions in heavy-ion collisions at various energies were shown to follow a curve in the temperature-baryo-chemical potential plane [35], which has been phenomenologically parametrized as [36]

$$
\begin{aligned}
T\left(\mu_{B}\right) & =a-b \mu_{B}^{2}-c \mu_{B}^{4}, \\
\mu_{B} & =d /\left(1+e \sqrt{s_{\mathrm{NN}}}\right),
\end{aligned}
$$

with $a=0.166 \pm 0.002 \mathrm{GeV}, b=0.139 \pm 0.016 \mathrm{GeV}^{-1}$, $c=0.053 \pm 0.021 \mathrm{GeV}^{-3}, d=1.308 \pm 0.028 \mathrm{GeV}$, and $e=$ $0.273 \pm 0.008 \mathrm{GeV}^{-1}$. This parametrization is quantitatively similar to the one proposed in Ref. [37] and results in a very satisfactory description of different particle excitation functions measured in nucleus-nucleus collisions.

The increase of the antimatter-to-matter ratio in heavy-ion collisions with the increase of center-of-mass energy has been observed by the NA49 $[38,39]$ and STAR [40] Collaborations. Figure 1 shows changes of the $\bar{p} / p$ ratio with collision energy at mid-rapidity in central heavy-ion and in $p+p$ collisions. The data from the NA49 and STAR Collaborations are compared with new results from the ALICE Collaboration [2]. There is a clear increase of this ratio toward unity, indicating
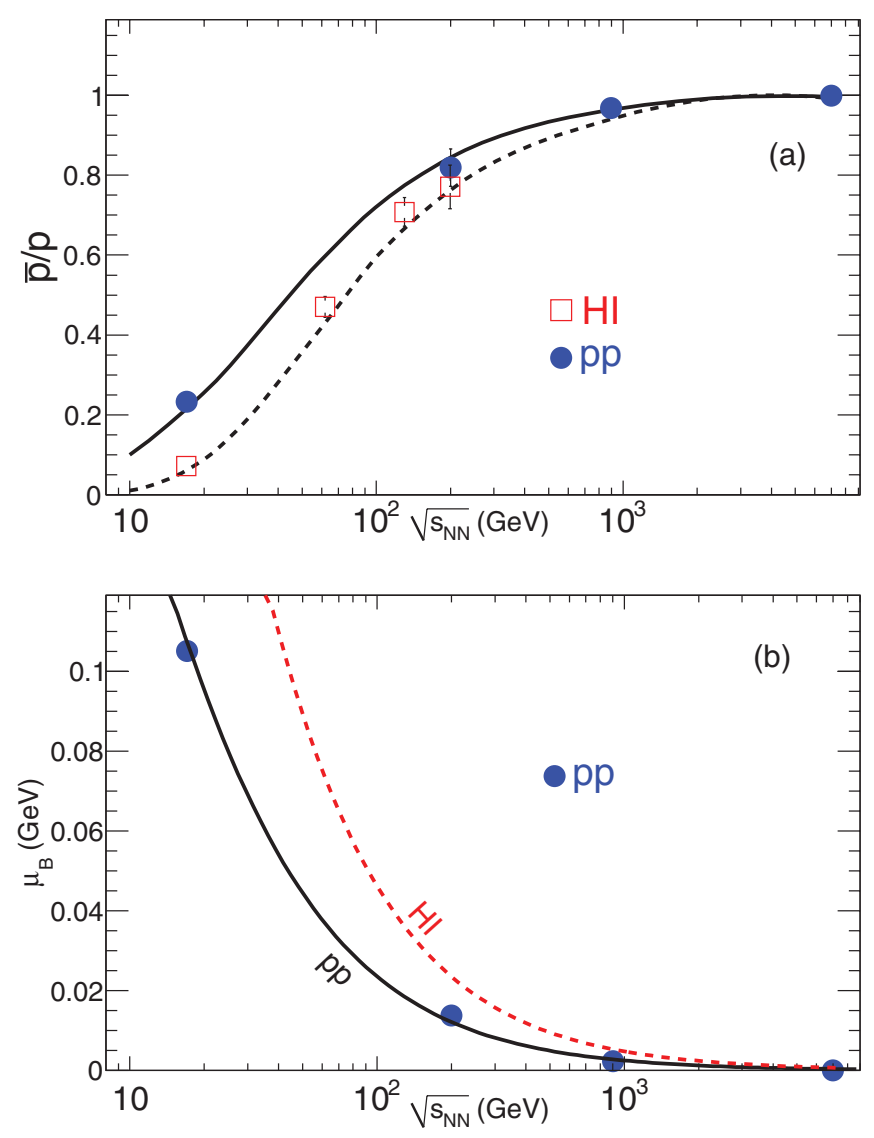

FIG. 1. (Color online) The $\bar{p} / p$ ratio (a) and the corresponding $\mu_{B}$ (b) as a function of $\sqrt{s_{\mathrm{NN}}}$. The solid circles are results from $p+p$ collisions and the open squares are results from heavy-ion collisions [1,2,38-40]. The dashed line is the parametrization for heavy-ion collisions from Ref. [36], while the solid line is the new parametrization for $p+p$ collisions. 
approximate symmetry of matter and antimatter at the LHC energy. There is also a clear increase of the $\bar{p} / p$ ratio when going from heavy-ion toward $p+p$ collisions.

In Fig. 1 data are compared with statistical-thermal model results. In heavy-ion collisions the energy dependencies of the model parameters were taken from Eqs. (2) and (3). There is a clear agreement of model predictions with data. For $p+p$ collisions no systematic analyses of model parameters versus energy were performed before now.

If (anti)nucleons are directly originating from a thermal source, then from Eq. (1) (i.e., neglecting feed-down from resonances) it is clear that the $\bar{p} / p$ densities ratio

$$
\frac{n_{\bar{p}}}{n_{p}}=\exp \left(-2 \mu_{B} / T\right)
$$

is quantified by the values of $\mu_{B} / T$. Thus, an increase in the $\bar{p} / p$ ratio from heavy-ion to $p+p$ collisions, seen in Fig. 1, is due to a decrease in the $\mu_{B} / T$ value.

To extract the corresponding $\mu_{B}$ and $T$ at fixed energy in $p+p$ collisions we have used the THERMUS code [28,29], which correctly accounts for feeding corrections to (anti)nucleons from decays of heavier resonances.

The $\bar{p} / p$ ratios measured in $p+p$ collisions, shown in Fig. 1, have been fitted using the statistical-thermal model by varying only the parameters $d$ and $e$ in Eqs. (2) and (3). We have used the same $T\left(\sqrt{s_{\mathrm{NN}}}\right)$ dependence for $p+p$ as for heavy-ion collisions. This is justified by the observation that at high energies there is no noticeable change in $T$ between central and peripheral heavy-ion collisions as well as $p+p$ collisions [41]. The resulting baryo-chemical potential $\mu_{B}$ is shown in the lower part of Fig. 1 by filled circles. In addition, applying the parametrization of $\mu_{B}\left(\sqrt{s_{\mathrm{NN}}}\right)$ as in Eq. (3),

$$
\mu_{B}=d_{\mathrm{pp}} /\left(1+e_{\mathrm{pp}} \sqrt{s_{\mathrm{NN}}}\right),
$$

we have found that, in $p+p$ collisions, $d_{\mathrm{pp}}=0.4 \mathrm{GeV}$ and $e_{\mathrm{pp}}=0.1599 \mathrm{GeV}^{-1}$. The solid line in the lower part of Fig. 1 shows the energy dependence of $\mu_{B}$ in $p+p$ collisions from Eq. (5). For comparison, also shown in this figure is the energy dependence of $\mu_{B}$ expected in heavy-ion collisions. From Fig. 1 it is clear that at mid-rapidity $\mu_{B}$ is always lower in $p+p$ than in heavy-ion collisions. This observation reflects the fact that at mid-rapidity the stopping power in $p+p$ collisions is less than in heavy-ion reactions. The change of the $\bar{p} / p$ ratio with energy in $p+p$ collisions is quantified in the upper part of Fig. 1 using the parametrization of $\mu_{B}\left(\sqrt{s_{\mathrm{NN}}}\right)$ from Eq. (5).

When neglecting feed-down contributions from resonances, the antibaryon/baryon ratio composed of $N_{S}$-strange quarks or antiquarks,

$$
\frac{n_{\bar{B}}}{n_{B}}=\exp \left[-2\left(\mu_{B}-N_{S} \mu_{S}\right) / T\right]
$$

is modified by the strange chemical potential. As $\mu_{S}$ is always smaller than $\mu_{B}$ (see, e.g., [24,42]), the above ratios should appear ordered with the strangeness quantum numbers; i.e., as $N_{S}$ increases, the difference between antibaryon and baryon yields decreases.

Figure 2 shows data on strange antibaryon/baryon ratios from SPS and RHIC energies compared with model
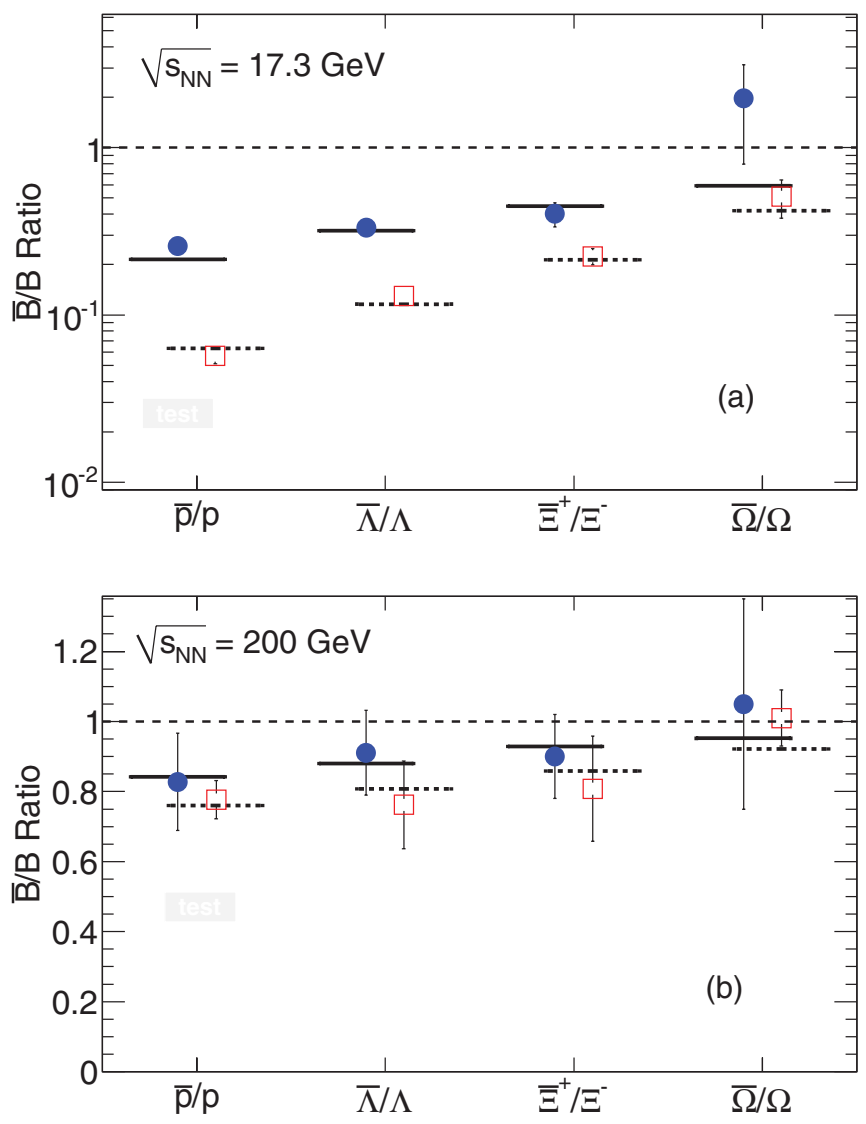

FIG. 2. (Color online) Antibaryon-to-baryon ratios sorted according to their strangeness content. Circles (solid horizontal line) refer to $p+p$ collision data (model calculations) and open squares (dashed horizontal line) refer to heavy-ion collision data (model calculations). (a) Results at SPS energy and (b) at RHIC energy.

calculations using the THERMUS code. The data and the model results in both $p+p$ and heavy-ion collisions are in good agreement. There are clear trends in strange antibaryon/baryon ratios already expected from the simplified Eq. (6). (i) With increasing strangeness quantum number, the antibaryon/baryon ratios are increasing and approaching unity. (ii) In heavy-ion collisions the $\bar{B} / B$ ratios are smaller than in $p+p$ collisions due to larger values of $\mu_{B}$. This is particularly evident at SPS energies, where the difference in $\mu_{B}$ in $p+p$ and $\mathrm{Pb}+\mathrm{Pb}$ systems is larger than at RHIC. (iii) The differences between heavy-ion and $p+p$ collisions decrease with increasing $\sqrt{s_{\mathrm{NN}}}$. At LHC energies the $\bar{p} / p$ ratio is close to unity, and therefore the abundances of strange and antistrange baryons should be similar.

\section{PRODUCTION OF (ANTI)NUCLEI AND (ANTI)HYPERNUCLEI}

\section{A. Comparison to data from RHIC}

The relative production of antimatter to matter, expressed by ratios of antibaryon/baryon yields, was shown in the last section to be well described by the statistical-thermal model. Thus it is of interest to verify whether the recently observed 
production of light (anti)nuclei including (anti)hypertritons $\left({ }_{\Lambda}^{3} \mathrm{H}\right)$ in heavy-ion collisions at RHIC [9] also follows a pattern expected in the statistical-thermal model.

Studying the antinuclei/nuclei ratio in the statistical-thermal model, we see that an extra factor of $\mu_{B}$ is added each time the baryon number is increased. Thus each nucleon adds a factor of $\mu_{B}$ in the exponent of the Boltzmann factor in Eq. (1). Therefore the production of nuclear fragments is very sensitive to the value of the baryo-chemical potential. Consequently the antinuclei/nuclei ratios are excellent observables for a precise determination of $\mu_{B}$.

Deuterium has an additional neutron and the antideuterium/deuterium ratio in the statistical-thermal model is given by

$$
\frac{n_{\bar{d}}}{n_{d}}=\exp \left(-4 \mu_{B} / T\right)
$$

By neglecting contributions of heavier resonances to nucleon yields, it should be similar to the square of the antiproton/proton ratio. ${ }^{3} \mathrm{He}$ has three nucleons and the corresponding anti- ${ }^{3} \mathrm{He} /{ }^{\beta} \mathrm{He}$ ratio is given by

$$
\frac{n_{3^{3} \mathrm{He}}}{n_{{ }^{3} \mathrm{He}}}=\exp \left(-6 \mu_{B} / T\right)
$$

which then is $\sim(\bar{p} / p)^{3}$.

If the nuclei carry strangeness, this leads to an extra term $\mu_{S}$ and the antihypertriton/hypertriton ratio reads

$$
\frac{n_{\bar{\Lambda}} \mathrm{H}}{n_{{ }_{\Lambda} \mathrm{H}}}=\exp \left[-\left(6 \mu_{B}-2 \mu_{S}\right) / T\right] \text {. }
$$

In mixed ratios, i.e., ratios of different nuclei (or antinuclei), there appears an extra factor due to different degeneracy and masses; e.g., in the statistical-thermal model,

$$
\frac{n_{{ }^{3}} \mathrm{H}}{n_{3} \mathrm{He}}=\frac{g_{\Lambda} \mathrm{H}}{g_{{ }^{3} \mathrm{He}}} \frac{\left(m_{{ }^{3} \mathrm{H}}\right)^{2}}{\left(m_{3} \mathrm{He}\right)^{2}} \frac{K_{2}\left(m_{{ }^{3}} \mathrm{H} / T\right)}{K_{2}\left(m_{3} \mathrm{He} / T\right)} \exp \left(-\mu_{S} / T\right) .
$$

Figure 3 shows comparisons of the statistical-thermal model results for different (anti)nuclei ratios with recent experimental data from the STAR Collaboration. The data on the ${ }^{3} \mathrm{He}$ and $\overline{{ }^{3} \mathrm{He}}$ yields have been corrected for contamination from hypertriton and antihypertriton decays, assuming a decay branching ratio of $25 \%$. Consequently, in the model calculations, such decays have not been included.

In the statistical-thermal model, following Eqs. (8) and (9), ratios of (anti)nuclei/nuclei are entirely quantified by the $\mu_{B} / T$ and $\mu_{S} / T$ values. From Fig. 3 it clear that, using the thermal parameters at chemical freeze-out obtained from the analysis of particle yields at RHIC, there is an excellent description of measured ratios of $\overline{{ }^{3} \mathrm{He}} /{ }^{3} \mathrm{He}$ and $\overline{{ }_{\Lambda}^{3} \mathrm{H}} /{ }_{\Lambda}^{3} \mathrm{H}$. However, deviations are seen on the level of the mixed ratios $\overline{{ }_{\Lambda}^{3} \mathrm{H}} \overline{{ }^{3} \mathrm{He}}$ and ${ }_{\Lambda}^{3} \mathrm{H} /{ }^{3} \mathrm{He}$.

In elementary collisions, nuclei and antinuclei as well as hypernuclei and antihypernuclei can be produced by direct pair production. In heavy-ion collisions, due to final-state correlations, a different production mechanism opens up through hadron coalescence. Indeed, production of nuclei in $\mathrm{Pb}+\mathrm{Pb}$ collisions at $\sqrt{s_{\mathrm{NN}}}=17.3 \mathrm{GeV}$ at the CERN SPS [43] has been found to be consistent with a coalescence picture,

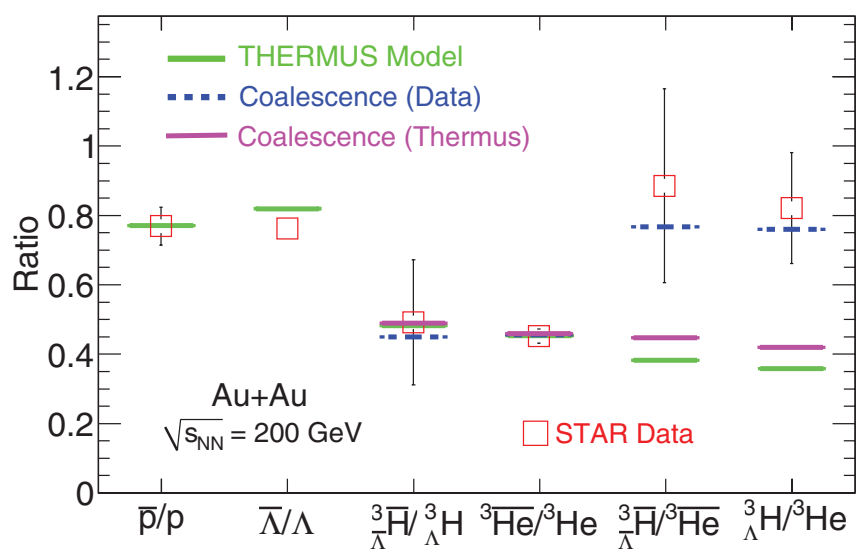

FIG. 3. (Color online) Comparison of results from the STAR Collaboration with the statistical-thermal and the coalescence models. For the latter, both experimental values [44,45] (dashed lines) and values from the statistical-thermal model (dash-dotted lines) have been used.

while this was not the case in $p+\mathrm{Be}$ collisions at the same energy.

In the most straightforward coalescence picture the ratios of different (anti)nuclei can be directly related to ratios of hadronic yields. In particular,

$$
\begin{aligned}
& \frac{\overline{{ }^{3} \mathrm{e}}}{{ }^{3} \mathrm{He}}=\frac{\overline{p p n}}{p p n} \simeq\left(\frac{\bar{p}}{p}\right)^{3}, \\
& \frac{\overline{{ }^{3} \mathrm{H}}}{\frac{\overline{p n} \Lambda}{p n}} \simeq\left(\frac{\bar{p}}{p}\right)^{2} \frac{\bar{\Lambda}}{\Lambda}, \\
& { }_{\Lambda}^{{ }_{\Lambda} \mathrm{H}}=\frac{\overline{p n \Lambda}}{{ }_{\Lambda}^{3} \mathrm{H}}=\frac{p n \Lambda}{p p n} \simeq \frac{\Lambda}{p},
\end{aligned}
$$

and

$$
\frac{\overline{{ }_{\Lambda} \mathrm{H}}}{\overline{{ }^{3} \mathrm{He}}}=\frac{\overline{p n} \bar{\Lambda}}{\overline{p p n}} \simeq \frac{\bar{\Lambda}}{\bar{p}} .
$$

Comparing results of the statistical-thermal model with the coalescence framework, one sees that some ratios are quite similar. Indeed, from Eqs. (8) and (11) as well as from Eqs. (9) and (12) it is clear that, by neglecting feed-down from resonance decays, the statistical-thermal model coincides with coalescence predictions for the $\overline{{ }^{3} \mathrm{He}} /{ }^{3} \mathrm{He}$ and ${ }_{\Lambda}^{\overline{3} \mathrm{H}} /{ }_{\Lambda}^{3} \mathrm{H}$ ratios (see also Ref. [30]). Thus, as long as the key input ratios $\bar{p} / p$ and $\bar{\Lambda} / \Lambda$ are in agreement with the thermal descriptions, the measured ratios do not allow one to distinguish between the two mechanisms. However, differences between these models are seen on the level of mixed ratios, $\overline{{ }_{\Lambda}^{3} \mathrm{H}} / \overline{{ }^{3} \mathrm{He}}$ and ${ }_{\Lambda}^{3} \mathrm{H} /{ }^{3} \mathrm{He}$, due to different masses of nuclei. From Eqs. (10) and (13) one finds that, when neglecting binding energy of nuclei and feed-down corrections, the statistical-thermal model differs from the coalescence framework by a factor of $\left(1 / 3+2 m_{p} / 3 m_{\Lambda}\right)^{3 / 2}$. Consequently, the statistical-thermal model results for the $\overline{{ }_{\Lambda}^{3} \mathrm{H}} / \overline{{ }^{3} \mathrm{He}}$ and ${ }_{\Lambda}^{3} \mathrm{H} /{ }^{3} \mathrm{He}$ ratios should be lower than those obtained in the coalescence picture.

The results from the coalescence model $[6,46]$ are compared to data from the STAR Collaboration and the 

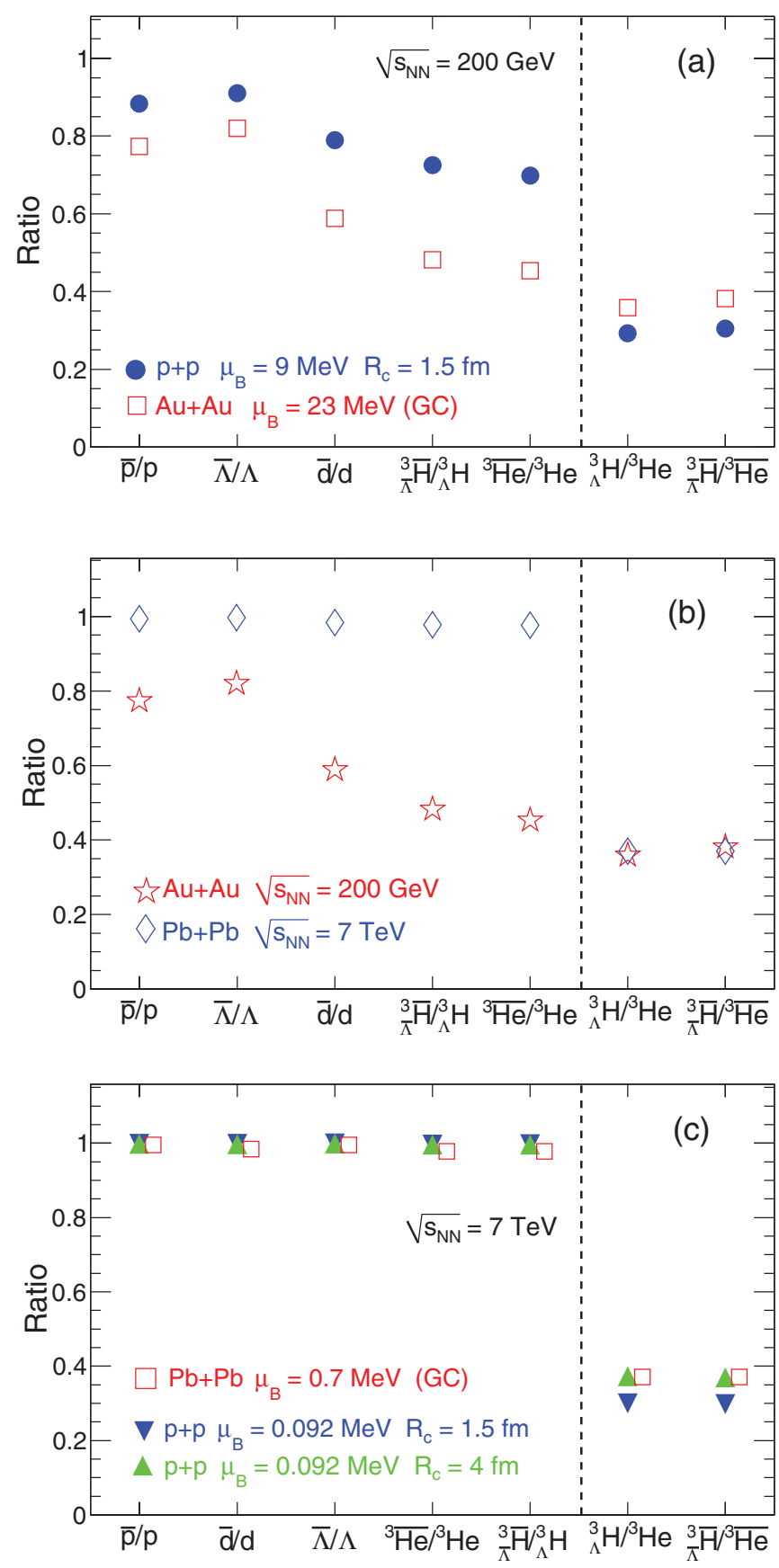

FIG. 4. (Color online) Comparison of different particle ratios calculated in the statistical-thermal model using $T=170 \mathrm{MeV}$. (a) Results for $p+p$ and heavy-ion collisions at $\sqrt{s_{\mathrm{NN}}}=200 \mathrm{GeV}$. (b) Results for heavy-ion collisions at different collision energies. (c) Prediction for $p+p$ and $\mathrm{Pb}+\mathrm{Pb}$ collisions at $\sqrt{s_{\mathrm{NN}}}=7 \mathrm{TeV}$.

statistical-thermal model predictions in Fig. 3. The coalescence estimate was obtained using the $\bar{p} / p, \bar{\Lambda} / \Lambda, \bar{\Lambda} / \bar{p}$, and $\Lambda / p$ ratios both measured by the STAR Collaboration $[9,44,45]$ and obtained from the THERMUS calculations.

We note that in the coalescence picture the equilibrium abundances of particle yields are not required. Consequently, (anti)nuclei produced from the off-equilibrium medium can lead to particle ratios being in agreement with the simple coalescence estimate discussed above. However, this is no longer the case for the statistical-thermal model, which requires statistical order of particle yields in the final state.

\section{B. Predictions for RHIC and LHC energies}

In the preceding section we concentrated on the statisticalthermal model description of (anti)matter production in heavyion collisions up to RHIC energies. In the following we extend our discussion to higher incident energies and quantify differences between $p+p$ and heavy-ion collisions.

In Fig. 4 we compare $p+p$ and heavy-ion collisions at both RHIC and LHC energies. For simplicity, we use $\sqrt{s_{\mathrm{NN}}}=$ $7 \mathrm{TeV}$ for the LHC energy in this figure. The values of the actual energies are given in Table I. In the context of the statistical-thermal model, the difference between these two colliding systems is caused by different values of $\mu_{B}$ and by the effect of canonical suppression in $p+p$ collisions. The ratios of antinuclei to nuclei without strangeness content are only affected by the baryo-chemical potential, which at mid-rapidity is smaller in $p+p$ than in heavy-ion collisions, as discussed earlier.

Figure 4(a) nicely demonstrates that with increasing mass the effect of $\mu_{B}$ becomes stronger, yet a strangeness content causes an opposite trend, as discussed earlier. The ratio of hypertriton to ${ }^{3} \mathrm{He}$ and the corresponding antimatter ratio show the effect of the canonical suppression reducing the yield of (anti)baryons carrying strangeness. For the chosen correlation volume with $R_{c}=1.5 \mathrm{fm}$, the difference is not dramatic but is very noticeable.

The effect of increasing collision energy is demonstrated in Fig. 4(b). Here, the differences between the antimatter/matter ratios in heavy-ion collisions at RHIC and LHC energies are essentially due to the decreasing value of $\mu_{B}$. At LHC energy, the chemical potential is smaller than $1 \mathrm{MeV}$, resulting in the antimatter/matter ratio being close to unity. The ratios of the (anti)hypernuclei/(anti- $)^{3} \mathrm{He}$ remain nearly unchanged from RHIC to LHC energies since here the effect of $\mu_{B}$ is only due to the strange chemical potential, which is small. These ratios are dominated by mass differences and degeneracy factors.

The expectations for LHC energies are studied in more detail in Fig. 4(c) by comparing $p+p$ and $\mathrm{Pb}+\mathrm{Pb}$

TABLE I. Particle ratios for $p+p$ and heavy-ion collisions. GC refers to grand canonical. The values of $\sqrt{s_{\mathrm{NN}}}$ and of the canonical radius $R_{c}$ are given.

\begin{tabular}{lccc}
\hline \hline Ratio & $\begin{array}{c}\mathrm{Au}+\mathrm{Au} \\
200 \mathrm{GeV} \\
\mathrm{GC}\end{array}$ & $\begin{array}{c}\mathrm{Pb}+\mathrm{Pb} \\
2.76 \mathrm{TeV} / 5.5 \mathrm{TeV} \\
\mathrm{GC}\end{array}$ & $\begin{array}{c}p+p \\
7 \mathrm{TeV}\end{array}$ \\
& 0.77 & $0.98 / 0.99$ & $R_{c}=4 \mathrm{fm} / 1.5 \mathrm{fm}$ \\
$\overline{\bar{p}} / p$ & 0.82 & $0.985 / 0.993$ & $0.996 / 0.996$ \\
$\bar{\Lambda} / \Lambda$ & $0.96 / 0.98$ & $0.997 / 0.997$ \\
$\bar{d} / d$ & 0.59 & $0.95 / 0.97$ & $0.99 / 0.99$ \\
$\frac{{ }_{\Lambda}^{3} \mathrm{H} /{ }_{\Lambda}^{3} \mathrm{H}}{{ }^{3} \mathrm{He} /{ }^{3} \mathrm{He}}$ & 0.48 & $0.94 / 0.97$ & $0.99 / 0.99$ \\
$\frac{{ }_{\Lambda}^{3} \mathrm{H} /{ }^{3} \mathrm{He}}{{ }_{\Lambda}^{3} \mathrm{H} /{ }^{3} \mathrm{He}}$ & 0.45 & $0.37 / 0.37$ & $0.37 / 0.30$ \\
$\bar{~}$ & 0.38 & $0.37 / 0.37$ & $0.37 / 0.30$ \\
\hline
\end{tabular}




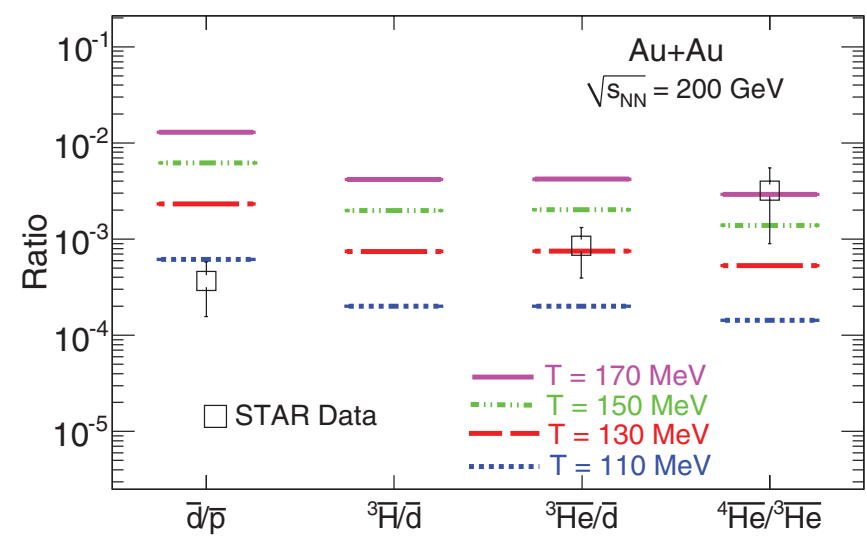

FIG. 5. (Color online) Comparison of model calculations of various nuclei ratios with different masses for $\mathrm{Au}+\mathrm{Au}$ collisions at $\sqrt{s_{\mathrm{NN}}}=200 \mathrm{GeV}$. The model results obtained with different freeze-out temperatures are compared with recently measured values $[3,47]$.

collisions. For simplicity, in both cases a collision energy of $7 \mathrm{TeV}$ has been chosen. The ratios do not change between $\sqrt{s_{\mathrm{NN}}}=2.76$ and $7 \mathrm{TeV}$. Table I gives the ratios for the energies under study at the LHC. The antimatter/matter ratios hardly change from $p+p$ to heavy-ion collisions. All antiparticle/particle ratios are close to unity. The ratios of (anti)hypernuclei/(anti-) $)^{3} \mathrm{He}$ exhibit the influence of canonical suppression for the correlation volume (see Sec. II) corresponding to $R_{c}=1.5 \mathrm{fm}$ [31]. For larger $R_{c}$ the canonical effect is reduced and already for $R_{c}=4 \mathrm{fm}$ can be neglected.

The predictions of the statistical-thermal model for ratios of particles with different masses are shown in Fig. 5. The calculations have been done for $\mathrm{Au}+\mathrm{Au}$ collisions at $\sqrt{s_{\mathrm{NN}}}=$ $200 \mathrm{GeV}$.

The thermal model results presented so far were obtained with the freeze-out temperature from Eqs. (2) and (3). It is clear that ratios of nuclei with different masses are strongly influenced by the value of the freeze-out temperature. Figure 5 shows the statistical-thermal model results obtained with $T$ varying between 110 and $170 \mathrm{MeV}$. These calculations are compared with recent data from the STAR Collaboration, which includes the observation of $\bar{\alpha}$ particles $[3,47]$. Figure 5 indicates that more data are needed before the freeze-out temperature for antinuclei can be deduced.

\section{SUMMARY}

We have discussed in a systematic manner the production of (anti)matter in high-energy collisions within the statisticalthermal model. We have focused on a general comparison of the production of antibaryons and antinuclei. The variation of $\bar{p} / p$ ratios with $\sqrt{s_{\mathrm{NN}}}$ being different for $p+p$ and heavy-ion collisions has been used to obtain the parametrization of the energy dependence of thermal parameters in $p+p$ collisions beyond the SPS energy. We have demonstrated the scaling behavior of (anti)baryon/baryon ratios with the strangeness quantum number and we have shown the changes in these ratios for $p+p$ and heavy-ion collisions with changing $\sqrt{s_{\mathrm{NN}}}$.

We have compared the measured ratios of nuclear and antinuclear fragments in heavy-ion collisions with the statisticalthermal model and with the coalescence model. Based on the successful description of existing data, we have made predictions for (anti)matter production in $p+p$ and heavy-ion collisions at LHC energies.

\section{ACKNOWLEDGMENTS}

We acknowledge support from the Deutsche Forschungsgemeinschaft (DFG), the Polish Ministry of Science (MEN), the Alexander von Humboldt Foundation, the ExtreMe Matter Institute (EMMI), the CNRS National Institute of Nuclear and Particle Physics (IN2P3), the University of Nantes, and the Ecoles des Mines. The financial support of the Federal Ministry of Education and Research (BMBF), the DFG-NRF Collaboration, the Department of Science and Technology of the Government of India, and the South Africa-Poland scientific collaboration is also gratefully acknowledged.
[1] B. I. Abelev et al. (STAR Collaboration), Phys. Rev. C 79, 034909 (2009).

[2] A. K. Aamodt et al. (ALICE Collaboration), Phys. Rev. Lett. 105, 072002 (2010).

[3] H. Agakishiev et al. (STAR Collaboration), Nature (London) 473, 353 (2011).

[4] M. Danysz and J. Pniewski, Philos. Mag. 44, 348 (1953).

[5] A. Kerman and M. Weiss, Phys. Rev. C 8, 408 (1973).

[6] H. Sato and K. Yazaki, Phys. Lett. B 98, 153 (1981).

[7] D. Hahn and H. Stoecker, Nucl. Phys. A 476, 718 (1988).

[8] H. Stoecker and W. Greiner, Phys. Rep. 137, 277 (1986).

[9] B. I. Abelev (STAR Collaboration), Science 328, 58 (2010).

[10] R. Rapp and E. V. Shuryak, Phys. Rev. Lett. 86, 2980 (2001).

[11] E. Fermi, Prog. Theor. Phys. 5, 570 (1950).

[12] I. Pomeranchuk, Dokl. Akad. Nauk Ser. Fiz. 78, 889 (1951).

[13] W. Heisenberg, Z. Phys. 133, 65 (1952).
[14] L. Landau, Izv. Akad. Nauk Ser. Fiz. 17, 51 (1953).

[15] R. Hagedorn, Nuovo Cimento Suppl. 3, 147 (1965).

[16] R. Hagedorn, Nuovo Cimento A 56, 1027 (1968).

[17] S. T. Butler and C. A. Pearson, Phys. Rev. 129, 836 (1963).

[18] A. Mekjian, Phys. Rev. Lett. 38, 640 (1977).

[19] H. G. Pugh, Quark Matter '84, edited by K. Kajantie, Lecture Notes in Physics (Springer Verlag, Berlin, 1984).

[20] F. Becattini and U. W. Heinz, Z. Phys. C 76, 269 (1997).

[21] P. Braun-Munzinger, K. Redlich, and J. Stachel, in Quark Gluon Plasma 3, edited by R. C. Hwa and Xin-Nian Wang (World Scientific, Singapore, 2003).

[22] F. Becattini, P. Castorina, J. Manninen, and H. Satz, Eur. Phys. J. C 56, 493 (2008).

[23] S. Kabana and P. Minkowski, New J. Phys. 3, 4 (2001).

[24] K. Redlich, J. Cleymans, H. Oeschler, and A. Tounsi, Acta Phys. Pol. B 33, 1609 (2002). 
[25] P. Braun-Munzinger, J. Cleymans, H. Oeschler, and K. Redlich, Nucl. Phys. A 697, 902 (2002).

[26] P. Braun-Munzinger and J. Stachel, Nucl. Phys. A 606, 320 (1996).

[27] F. Karsch and K. Redlich, Phys. Lett. B 695, 136 (2011).

[28] S. Wheaton and J. Cleymans, J. Phys. G 31, S1069 (2005).

[29] S. Wheaton, J. Cleymans, and M. Hauer, Comput. Phys. Commun. 180, 84 (2009).

[30] A. Andronic, P. Braun-Munzinger, J. Stachel, and H. Stoecker, Phys. Lett. B 697, 203 (2011).

[31] S. Hamieh, K. Redlich, and A. Tounsi, Phys. Lett. B 486, 61 (2000).

[32] R. Hagedorn and K. Redlich, Z. Phys. C 27, 541 (1985).

[33] J. Rafelski and M. Danos, Phys. Lett. B 97, 279 (1980).

[34] J. Cleymans, H. Oeschler, and K. Redlich, Phys. Rev. C 59, 1663 (1999).

[35] J. Cleymans and K. Redlich, Phys. Rev. Lett. 81, 5284 (1998).

[36] J. Cleymans, H. Oeschler, K. Redlich, and S. Wheaton, Phys. Rev. C 73, 034905 (2006).
[37] A. Andronic, P. Braun-Munzinger, and J. Stachel, Nucl. Phys. A 772, 167 (2006).

[38] C. Alt et al. (NA49 Collaboration), Phys. Rev. C 73, 044910 (2006).

[39] C. Alt et al. (NA49 Collaboration), Phys. Rev. C 77, 024903 (2008).

[40] B. I. Abelev et al. (STAR Collaboration), Phys. Rev. C 75, 064901 (2007).

[41] I. Kraus, J. Cleymans, H. Oeschler, K. Redlich, and S. Wheaton, Phys. Rev. C 76, 064903 (2007).

[42] J. Cleymans and H. Satz, Z. Phys. C 57, 135 (1993).

[43] R. Arsenescu et al. (NA52 Collaboration), New J. Phys. 5, 150 (2003).

[44] B. I. Abelev et al. (STAR Collaboration), Phys. Rev. Lett. 97, 152301 (2006).

[45] J. Adams et al. (STAR Collaboration), Phys. Rev. Lett. 98, 062301 (2007).

[46] B. L. Ioffe, I. A. Shushpanov, and K. N. Zyablyuk, Phys. At. Nucl. 68, 326 (2005).

[47] C. Adler et al. (STAR Collaboration), Phys. Rev. Lett. 87, 262301 (2001). 